\title{
CORRIGENDUM TO: "ON THE CIRCLE CRITERION FOR BOUNDARY CONTROL SYSTEMS IN FACTOR FORM: LYAPUNOV STABILITY AND LUR'E EQUATIONS"
}

\author{
Piotr Grabowski ${ }^{1}$ And Frank M. Callier ${ }^{2}$
}

\begin{abstract}
A corrected version of [P. Grabowski and F.M. Callier, ESAIM: COCV 12 (2006) 169-197], Theorem 4.1, p. 186, and Example, is given.
\end{abstract}

Mathematics Subject Classification. 93B, 47D, 35A, 34G.

Received September 8, 2008.

Published online June 18, 2009.

\section{INTRODUCTION}

The authors are deeply indebted to Hartmut Logemann, Department of Mathematics, University of Bath, UK for pointing out a counterexample, repeated below, showing that the statement of [2], Theorem 4.1, p. 186, is wrong.

With the notation of [2] all assumptions of that theorem are met for

$$
\mathrm{H}=\mathbb{R}, \quad A=-1=A^{-1}, h=-1\left(\Longleftrightarrow c^{\#} x=x\right), d=1, \quad \delta=1, e=\frac{8}{3}, q=\frac{16}{3},
$$

however the system $(3.1)$ has exactly two solutions $(\mathcal{H}, \mathcal{G})=\left(-\frac{8}{3}, 0\right),(\mathcal{H}, \mathcal{G})=\left(-\frac{2}{3}, 2\right)$ and none of them is such that $\mathcal{H} \geq 0$. This counterexample demonstrates that the assumptions of [2], Theorem 4.1, p. 186, are not enough to ensure non-negativity of $\mathcal{H}$.

The aim of this note is to correct the result by adding reasonable and non-restrictive assumptions which can be verified without solving (3.1) explicitly.

\section{Corrigendum of [2], Theorem 4.1 (I), P. 186}

Theorem 2.1. Let assumptions $(\mathbf{H 1})-(\mathbf{H 5})$ hold. Moreover assume that:

(H6) The operator $A:(D(A) \subset H) \longrightarrow H$ is such that the semigroup generated by $A^{-1}$ is $\mathbf{A S}$.

\footnotetext{
Keywords and phrases. Infinite-dimensional control systems, semigroups, Lyapunov functionals, circle criterion.

${ }^{1}$ Institute of Automatics, AGH University of Science and Technology, avenue A. Mickiewicz 30, B1, rm. 314, 30-059 Cracow,

Poland. pgrab@ia.agh.edu.pl

${ }^{2}$ University of Namur (FUNDP), Department of Mathematics, Rempart de la Vierge 8, 5000 Namur, Belgium.
} 
Then:

(i) The system (3.1) has a solution $(\mathcal{H}, \mathcal{G}), \mathcal{H} \in \mathbf{L}(H), \mathcal{H}=\mathcal{H}^{*} \geq 0$, provided that if $q>0$ then, in addition, the assumption (A3) holds and

$$
\frac{1}{1+\mu_{0} \hat{g}} \in H^{\infty}\left(\mathbb{C}^{+}\right) \quad \text { for } \quad \mu_{0}:=\frac{k_{1}+k_{2}}{2}
$$

$\mathcal{G} \in H$, where in particular: $\mathcal{G}$ is the solution of the realization equation (4.4), where $\phi$ is the spectral factor of the Popov function $\pi$ (given by (4.2)) such that $\phi(0)=\sqrt{\delta}$, and both $\phi$ and $1 / \phi$ are in $H^{\infty}\left(\mathbb{C}^{+}\right)$.

Remark 2.1. It should be emphasized that if $q \leq 0$ the statement of [2], Theorem 4.1(i), p. 186, is fully correct, i.e., the assertion holds without (A3) and (2.1). The claim [2], Theorem 4.1(ii), p. 186, does not require any correction.

Proof. The whole reasoning of the existing proof remains correct after removing: the sentence starting from the words: "The symbol of the Toeplitz operator ...", the footnote on p. 186 and after dropping the inequality $\mathcal{H} \geq 0$ in the sentence just following (4.17). Having this done, we may correct the proof as follows. Since $X$ is a solution of (4.15) given by (4.10) it is clear that

$$
\mathcal{H}=-X=\psi^{*}\left[(q \mathbb{F}-e I) \mathcal{R}^{-1}(q \mathbb{F}-e I)^{*}-q I\right] \psi \geq 0
$$

if $q \leq 0$, whence the claim of the remark above is met.

Now, consider the case $q>0\left(\Longrightarrow \mu_{0} \neq 0\right)$ where, in addition (A3) (i.e., $d$ is an admissible factor control vector) and (2.1) hold. Observe that

$$
1-\mu_{0} \underbrace{c^{\#} d}_{=-\hat{g}(0)} \neq 0
$$

for if not, by (4.2), we would have $\pi(0)=\delta=\left(1-\frac{k_{1}}{\mu_{0}}\right)\left(1-\frac{k_{2}}{\mu_{0}}\right)=-\left(\frac{k_{2}-k_{1}}{k_{1}+k_{2}}\right)^{2}<0$, which contradicts (4.3). Since the LHS of (2.2) satisfies the Riccati equation

$$
\left(A^{-1}\right)^{*} \mathcal{H}+\mathcal{H} A^{-1}+\underbrace{\left[\frac{1}{\sqrt{\delta}}(-\mathcal{H} d+e h)\right]}_{=-\mathcal{G}}\left[\frac{1}{\sqrt{\delta}}(-\mathcal{H} d+e h)\right]^{*}-q h h^{*}=0
$$

then, adding $\frac{\mu_{0}}{1-\mu_{0} c^{\#} d} h d^{*} \mathcal{H}+\frac{\mu_{0}}{1-\mu_{0} c^{\#} d} \mathcal{H} d h^{*}$ to both sides of $(2.3)$, we conclude that $\mathcal{H}$ satisfies the Lyapunov operator equation

$$
\left[A^{-1}+\frac{\mu_{0}}{1-\mu_{0} c^{\#} d} d h^{*}\right]^{*} \mathcal{H}+\mathcal{H}\left[A^{-1}+\frac{\mu_{0}}{1-\mu_{0} c^{\#} d} d h^{*}\right]=-\left(\mathcal{G}-q_{1} h\right)\left(\mathcal{G}-q_{1} h\right)^{*}-q_{0} h h^{*}
$$

with

or equivalently,

$$
q_{1}:=\frac{\mu_{0} \sqrt{\delta}}{1-\mu_{0} c^{\#} d}, \quad q_{0}=\frac{\left(k_{2}-k_{1}\right)^{2}}{4\left(1-\mu_{0} c^{\#} d\right)^{2}}>0
$$

$$
\left\langle A_{0} x, \mathcal{H} x\right\rangle_{\mathrm{H}}+\left\langle\mathcal{H} x, A_{0} x\right\rangle_{\mathrm{H}}=-\left[\left(\mathcal{G}-q_{1} h\right)^{*} A_{0} x\right]^{2}-q_{0}\left[h^{*} A_{0} x\right]^{2} \quad \forall x \in D\left(A_{0}\right),
$$

where

$$
A_{0} x:=A\left(x-\mu_{0} d c^{\#} x\right), \quad D\left(A_{0}\right)=\left\{x \in D\left(d^{*}\right): x-\mu_{0} d c^{\#} x \in D(A)\right\}
$$


This is because $A_{0}^{-1}=A^{-1}+\frac{\mu_{0}}{1-\mu_{0} c^{\#} d} \mathrm{~d} h^{*} \in \mathbf{L}(\mathrm{H})$. The operator $A_{0}$ arises by applying negative linear feedback $u=-\mu_{0} y$ to

$$
\left\{\begin{aligned}
\dot{x} & =A(x+u d) \\
y & =c^{\#} x
\end{aligned}\right\}
$$

and it corresponds to the Lur'e control system of [2], Figure 1.1, p. 170, with $f(y)=\mu_{0} y$. Since $c^{\#}$ is admissible and $\hat{g} \in \mathrm{H}^{\infty}\left(\mathbb{C}^{+}\right)$, for $\mathrm{L}^{2}(0, \infty)$-controls the output is given by

$$
y=\bar{P} x_{0}+\overline{\mathbb{F}} u
$$

where $\bar{P}$ and $\overline{\mathbb{F}}$ stand for the extended observability map and the extended input-output operator, both associated with (2.5). Thus, for the closed-loop system, by the Paley-Wiener theory, one has

$$
\left(I+\mu_{0} \overline{\mathbb{F}}\right) y=\bar{P} x_{0} \Longleftrightarrow\left(1+\mu_{0} \hat{g}\right) y=\widehat{\bar{P} x_{0}},
$$

and, due to (2.1), the last equation has a unique solution $\hat{y} \in \mathrm{H}^{2}\left(\mathbb{C}^{+}\right)$. Via the feedback law equation $u=-\mu_{0} y$ this implies that for any $x_{0}: u \in \mathrm{L}^{2}(0, \infty)$. Now [2], Lemma 2.11, p. 177, implies that for every initial condition $x_{0}$ the first equation of (2.5) has a unique weak solution, whence, by Ball's theorem [1], p. 371 (see also [4], p. 259), the operator $A_{0}$ generates a $\mathrm{C}_{0}$-semigroup $\left\{S_{0}(t)\right\}_{t \geq 0}$ on $\mathrm{H}$ which is $\mathbf{A S}$.

Now, for every $x_{0} \in D\left(A_{0}\right)$ and each $t \geq 0,(2.4)$ yields

$$
\frac{\mathrm{d}}{\mathrm{d} t}\left\langle S_{0}(t) x_{0}, \mathcal{H} S_{0}(t) x_{0}\right\rangle_{\mathrm{H}}=-\left[\left(\mathcal{G}-q_{1} h\right)^{*} A_{0} S_{0}(t) x_{0}\right]^{2}-q_{0}\left[h^{*} A_{0} S_{0}(t) x_{0}\right]^{2} .
$$

Integrating both sides from 0 to $t$ and employing $\mathbf{A S}$ we obtain

$$
\left\langle x_{0}, \mathcal{H} x_{0}\right\rangle_{\mathrm{H}}=\int_{0}^{\infty}\left\{\left[\left(\mathcal{G}-q_{1} h\right)^{*} A_{0} S_{0}(t) x_{0}\right]^{2}+q_{0}\left[h^{*} A_{0} S_{0}(t) x_{0}\right]^{2}\right\} \mathrm{d} t \geq 0 \quad \forall x_{0} \in D\left(A_{0}\right) .
$$

Since $D\left(A_{0}\right)$ is dense in $\mathrm{H}$ as a $\mathrm{C}_{0}$-semigroup generator and $\mathcal{H}=\mathcal{H}^{*} \in \mathbf{L}(\mathrm{H})$ we get $\mathcal{H} \geq 0$.

Remark 2.2. The above proof may be slightly, but not essentially, modified by concluding AS of the semigroup $\left\{\mathrm{e}^{t A_{0}^{-1}}\right\}_{t \geq 0}$ from the reciprocal system

$$
\left\{\begin{aligned}
\dot{x} & =A^{-1} x+u d \\
y & =-h^{*} x
\end{aligned}\right\}
$$

with the feedback law $u=-\frac{\mu_{0}}{1-\mu_{0} c^{\#} d} y$, with an aid of [3], Lemma 12, p. 959. This is possible if $d$ is admissible with respect to $\left\{\mathrm{e}^{t A^{-1}}\right\}_{t \geq 0}$ and $u \in \mathrm{L}^{2}(0, \infty)$ for any initial condition $x_{0} \in \mathrm{H}$. It is not difficult to see, using duality between observation and control (see [2], p. 173) and the arguments which led to [2], Lemma 2.6, p. 174, that the first condition holds iff $d$ is admissible. Since in the frequency-domain the closed-loop output equation reads as

$$
\begin{aligned}
\hat{y}(s) & =-h^{*}\left(s I-A^{-1}\right)^{-1} x_{0}-h^{*}\left(s I-A^{-1}\right)^{-1} d\left[-\frac{\mu_{0}}{1-\mu_{0} c^{\#} d} \hat{y}(s)\right] \\
& =\left(U \widehat{\bar{P} x_{0}}\right)(s)+G(s)\left[-\frac{\mu_{0}}{1-\mu_{0} c^{\#} d}\right] \hat{y}(s),
\end{aligned}
$$

where $U$ is the unitary operator introduced in [2], p. 174, and $G$ is given by [2], (4.12), p. 187, then the second condition holds if $\frac{1}{1+\frac{\mu_{0}}{1-\mu_{0} c^{\#} d} G} \in \mathrm{H}^{\infty}\left(\mathbb{C}^{+}\right)$. By [2], (4.13), p. 187, the last condition is equivalent to (2.1). 
Next, our Lyapunov operator equation

$$
\left(A_{0}^{-1}\right)^{*} \mathcal{H}+\mathcal{H} A_{0}^{-1}=-\left(\mathcal{G}-q_{1} h\right)\left(\mathcal{G}-q_{1} h\right)^{*}-q_{0} h h^{*}
$$

allows to get directly

$$
\left\langle x_{0}, \mathcal{H} x_{0}\right\rangle_{\mathrm{H}}=\int_{0}^{\infty}\left\{\left[\left(\mathcal{G}-q_{1} h\right)^{*} \mathrm{e}^{t A_{0}^{-1}} x_{0}\right]^{2}+q_{0}\left[h^{*} \mathrm{e}^{t A_{0}^{-1}} x_{0}\right]^{2}\right\} \mathrm{d} t \geq 0 \quad \forall x_{0} \in \mathrm{H} .
$$

\section{Correction of [2], Example}

Just before the sentence starting from the words ([2], Sect. 5.2, p. 1927): "Thus all assumptions of Theorem 4.1 are met ..." the following text should be inserted ${ }^{3}$.

Recall that $d$ is an admissible factor control vector and for $b \in(0,1)$ the assumption (2.1) holds. Indeed, here

$$
\frac{1}{1+\mu_{0} \hat{g}(s)}=\frac{1}{1+\frac{4 b}{a(1+b)} \frac{a \mathrm{e}^{-s r}}{1+b \mathrm{e}^{-2 s r}}}=\frac{1+b \mathrm{e}^{-2 s r}}{b \mathrm{e}^{-2 s r}+\frac{4 b}{(1+b)} \mathrm{e}^{-s r}+1} .
$$

The numerator is bounded by $1+b$ on $\overline{\mathbb{C}^{+}}$, while for the denominator one has

$$
b \mathrm{e}^{-2 s r}+\frac{4 b}{(1+b)} \mathrm{e}^{-s r}+1=b\left(z_{0}-\mathrm{e}^{-s r}\right)\left(\overline{z_{0}}-\mathrm{e}^{-s r}\right), \quad \operatorname{Re} z_{0}=\frac{-2 b}{1+b}, \quad\left|z_{0}\right|^{2}=\frac{1}{b},
$$

whence

$$
\left|b \mathrm{e}^{-2 s r}+\frac{4 b}{(1+b)} \mathrm{e}^{-s r}+1\right|=b\left|z_{0}-\mathrm{e}^{-s r}\right|\left|\overline{z_{0}}-\mathrm{e}^{-s r}\right| \geq b\left(\left|z_{0}\right|-1\right)^{2}=(1-\sqrt{b})^{2},
$$

and consequently: $\left\|\frac{1}{1+\mu_{0} \hat{g}}\right\|_{\mathrm{H}^{\infty}\left(\mathbb{C}^{+}\right)} \leq \frac{1+b}{(1-\sqrt{b})^{2}}<\infty$.

\section{REFERENCES}

[1] J.M. Ball, Strongly continuous semigroups, weak solutions, and the variation of constants formula. P. Am. Math. Soc. 63 (1977) 370-373.

[2] P. Grabowski and F.M. Callier, On the circle criterion for boundary control systems in factor form: Lyapunov stability and Lur'e equations. ESAIM: COCV 12 (2006) 169-197.

[3] J.C. Oostveen and R.F. Curtain, Riccati equations for strongly stabilizable bounded linear systems. Automatica 34 (1998) 953-967.

[4] A. Pazy, Semigroups of Linear Operators and Applications to Partial Differential Equations. Springer Verlag, New York (1983).

\footnotetext{
${ }^{3}$ Since $q=k_{1} k_{2}<0$ for $b \in(0,3-2 \sqrt{2})$ and sufficiently small $\nu$ then, in fact, corrections are needed only for $b \in[3-2 \sqrt{2}, 1)$.
} 